\title{
The effect of menopause on the sexual dimorphism in the human retina - texture analysis of optical coherence tomography data ${ }^{\star}$
}

\author{
Ana Nunes ${ }^{1[0000-0001-5821-7964]}$, Pedro Serranho ${ }^{1,2}[0000-0003-2176-3923]$, Hugo \\ Quental ${ }^{1[0000-0001-9504-6543]}$, Miguel Castelo-Branco ${ }^{1,3[0000-0003-4364-6373]}$, \\ and Rui Bernardes ${ }^{1,3}[0000-0002-6677-2754]$ \\ 1 Univ Coimbra, Coimbra Institute for Biomedical Imaging and Translational \\ Research (CIBIT), Institute of Nuclear Sciences Applied to Health (ICNAS) \\ ${ }^{2}$ Universidade Aberta, Department of Sciences and Technology \\ 3 Univ Coimbra, Coimbra Institute for Biomedical Imaging and Translational \\ Research (CIBIT), Faculty of Medicine (FMUC) \\ Corresponding author: R. Bernardes - rmbernardes@fmed.uc.pt
}

\begin{abstract}
Sexual dimorphism in the human retina has recently been connected to gonadal hormones. In the study herein presented, texture analysis was applied to computed mean value fundus (MVF) images from optical coherence tomography data of female and male healthy adult controls. Two separate age-group analyses that excluded the probable perimenopause period of the women in the present study were performed, using a modified MVF image computation method that further highlights texture differences present in the retina. While distinct texture characteristics were found between premenopausal females and age-matched males, these differences almost disappeared in the older groups (postmenopausal women vs age-matched men), suggesting that sex-related texture differences in the retina can be correlated to the hormonal changes that women go through during the menopausal transition. These findings suggest that texture-based metrics may be used as biomarkers of physiology and pathophysiology of the retina and the central nervous system.
\end{abstract}

Keywords: texture analysis · optical coherence tomography · retina · healthy controls $\cdot$ sexual dimorphism $\cdot$ menopause

\section{Introduction}

Sexual dimorphism is a well-known phenomenon, manifested at different organ systems. In the visual system, sex-based differences exist both in the physiology and in the pathology of the eye and, more specifically, the retina 26. Sex-based

\footnotetext{
* This study was supported by The Portuguese Foundation for Science and Technology (PEst-UID/NEU/04539/2019 and UID/04950/2017), by FEDER-COMPETE (POCI-01-0145-FEDER-007440 and POCI01-0145-FEDER-016428), and by Centro 2020 FEDER-COMPETE (BIGDATIMAGE, CENTRO-01-0145-FEDER-000016).
} 
differences in the human retina have been extensively reported for the full retinal thickness 2 20|17/19]32 35] and less often, for the different retinal layers [27. Furthermore, there is a link between sex and retinal disorders, which has been attributed to gonadal hormones [26. Importantly, these hormones have a significant role in neurological disorders, such as Alzheimer's disease (AD) 28, which are closely related to the physiological processes within the retina, given the well-established relationship between the retina and the central nervous system (CNS) 2133.

Optical coherence tomography (OCT) studies addressing the sex-related differences in the retina rely mostly on layer thickness measurements. While most of these studies report thickness differences amongst the retinas of female and male healthy individuals [2]9/7/19|27/32]35, inconclusive results [613] have been reported as well. One limitation of these studies that may be influencing the sexrelated differences found (or lack thereof) is the typical inclusion of study groups with a wide age range and an unbalanced female-to-male ratio. On the other hand, the use of thickness as the metric of choice in these studies is somewhat restrictive, as new methodologies, notably the ones using texture analysis [25|3], have shown to reveal information not conveyed by thickness.

Recently, our group has been applying texture analysis to OCT data to gain further insight on the differences in the retinal structure, between distinct healthy control groups 23] and between healthy individuals and patients diagnosed with several neurodegenerative disorders, namely Alzheimer's and Parkinson's diseases [25] and multiple sclerosis [24]. Furthermore, the same analysis methodology was applied to animal studies, in which OCT data from a mouse model of $\mathrm{AD}$ and control mice groups were examined [22]10. These studies further confirmed the power of texture analysis to unveil information from OCT data, yet unexplored.

The present study constitutes a follow-up analysis of previous work 23, where texture features were computed from OCT data of female and male healthy adult controls, to identify differences in the retina associated with the subjects sex. In the work herein presented, the same texture analysis methodology was applied to a larger population. Furthermore, this population was split into younger and older age-groups, leaving out females at the menopausal transition period. As the sexual dimorphism in both the healthy and the diseased retina is correlated to hormonal differences [26], the goal of this grouping of the subjects in the present study is to examine how the cessation of menstrual cycles manifests in the retina, namely by analysing its impact on the previously identified sex-based differences 23. Also, this study aims to assess the impact of two distinct methods to compute fundus images for each retinal layer in spotting texture differences. 


\section{Material and Methods}

\subsection{Participants}

Data from 100 healthy controls, 50 females and 50 males, with no previous retinal pathologies, were gathered from the authors institutional database. The data collection protocol used for gathering OCT data was approved by the Ethics Committee of the Faculty of Medicine of the University of Coimbra and performed according to the tenets stated in the Declaration of Helsinki [36].

Female subjects were split into two groups: one younger, premenopausal group (30 women) and one older, postmenopausal group (20 women). As no information regarding the phase of the menstrual cycle/onset of menopausal transition at the time of image acquisition was available for any of the females in the present study, the median age of menopause, 51 years old [12, was the reference used. To exclude the probable perimenopause period, and maximise the number of included subjects amongst the ones available in the authors' institutional database, women aged 42-54 years old were not considered for analysis.

Male subjects were selected to ensure an exact age-match to the female subjects, where possible. All females and males were perfectly age-matched for the younger groups, while for the older groups, 7 out of 20 female/male pairs (35\%) were not perfectly age-matched.

Both eyes of each subject were analysed, except for one right eye from the younger female group, one right eye from the older female group, and one left eye from the older male group, which were excluded due to scan quality, yielding a total of 197 eye scans. Demographic data for the four groups in the present study are shown in table 1 .

Table 1. Demographic data of the groups at study.

\begin{tabular}{|l|c|c|c|c|c|}
\hline Group & $\mathrm{N}$ & $\begin{array}{c}\text { Age (years) } \\
\text { Mean(STD) }\end{array}$ & $\begin{array}{c}\text { Age (years) } \\
\text { Min(Max) }\end{array}$ & $\begin{array}{c}\text { Eyes } \\
\text { Right(Left) }\end{array}$ & \# Eye scans \\
\hline Younger Females & 30 & $30(6.9)$ & $19(42)$ & $29(30)$ & 59 \\
Younger Males & 30 & $30(6.9)$ & $19(42)$ & $30(30)$ & 60 \\
Older Females & 20 & $64(5.3)$ & $54(74)$ & $19(20)$ & 39 \\
Older Males & 20 & $68(8.8)$ & $54(79)$ & $20(19)$ & 39 \\
\hline
\end{tabular}

\subsection{OCT imaging}

The Cirrus SD-OCT 5000 (Carl Zeiss Meditec, Dublin, CA, USA) was used to gather all eye scans. The $512 \times 128$ Macular Cube protocol was used, centred on the macula. 


\subsection{Image processing}

OCT data were processed using the OCT Explorer software (Retinal Image Analysis Lab, Iowa Institute for Biomedical Imaging, Iowa City, IA, USA) [20|111] to segment the six innermost layers of the retina: the retinal nerve fibre layer (RNFL), the ganglion cell layer (GCL), the inner plexiform layer (IPL), the inner nuclear layer (INL), the outer plexiform layer (OPL) and the outer nuclear layer (ONL).

For each of the six layers at study, a mean value fundus (MVF) image was computed where each pixel is the average of the A-scan zeroing all A-scan values outside the layer of interest (see figure 1). This MVF image computation method is a modified approach based on the method originally developed by our group 14], where each pixel in the image is computed as the average of the A-scan values within the respective layer. The rationale for the use of the alternative MVF method is described in section 2.5.

Fig. 1. Colour-coded MVF images from the right eye of a male healthy control, in which each pixel is the average of the corresponding A-scan zeroing all A-scan values outside the layer of interest. From left to right and top to bottom: RNFL, GCL, IPL, INL, OPL and ONL layer fundus images. For reference purposes only: images were intensity-corrected, pseudo-colour coded and downsampled to $128 \times 128$, for ease of visualisation.

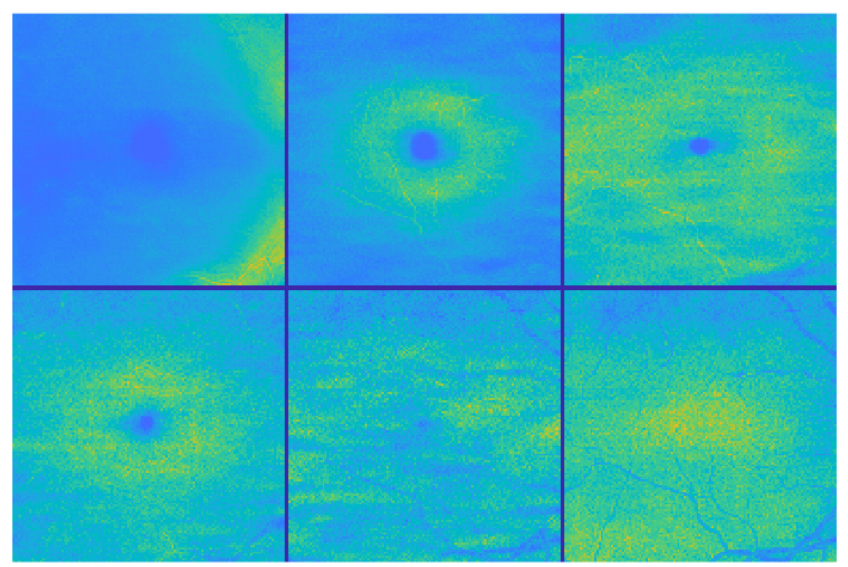

As both eyes from each subject were considered for analysis, all left-eye MVF images were horizontally flipped to match the temporal and nasal regions across all eye scans. 


\subsection{Texture analysis}

Texture features were computed for each MVF image, following an approach used previously [25124]. Two different texture analysis approaches were considered: the grey-level co-occurrence matrix (GLCM) [16], used to highlight local intensity variations, and the dual-tree complex wavelet transform (DTCWT) [30, used to identify coarser texture properties [18].

For the GLCM analysis, each image was down-sampled to $128 \times 128$ pixels and split into $7 \times 7$ blocks (see figure 2), which were independently analysed, except for the blocks in the central $\left(4^{\text {th }}\right)$ row and column, which were not considered to exclude the foveal region. For each block, four GLCMs were computed for distinct pixel pair orientations $\left(0^{\circ}, 45^{\circ}, 90^{\circ}\right.$, and $\left.135^{\circ}\right)$, using the distance of one pixel and considering $180^{\circ}$ apart angles to be the same. For each block and orientation, 20 features (defined in [161518131/7) were computed. The maximum value across the four orientations was selected as the feature value for each block. Blocks were then aggregated into quadrants: the temporal-superior (Q1), nasal-superior (Q2), temporal-inferior (Q3) and nasal-inferior (Q4) quadrants, composed of $3 \times 3$ blocks each (figure 2 . The average feature value, across the $3 \times 3$ blocks, was used as the final quadrant feature value.

Fig. 2. Computed fundus image from the volumetric macular cube scan of the right eye of a healthy control subject [25]. Each of the $7 \times 7$ blocks show the individually analysed areas which results were later aggregated into larger regions (shaded areas). Image axes are: $\mathrm{x}$-axis (horizontal) - temporal (left) to nasal (right) and y-axis(vertical) superior (top) to inferior (bottom).

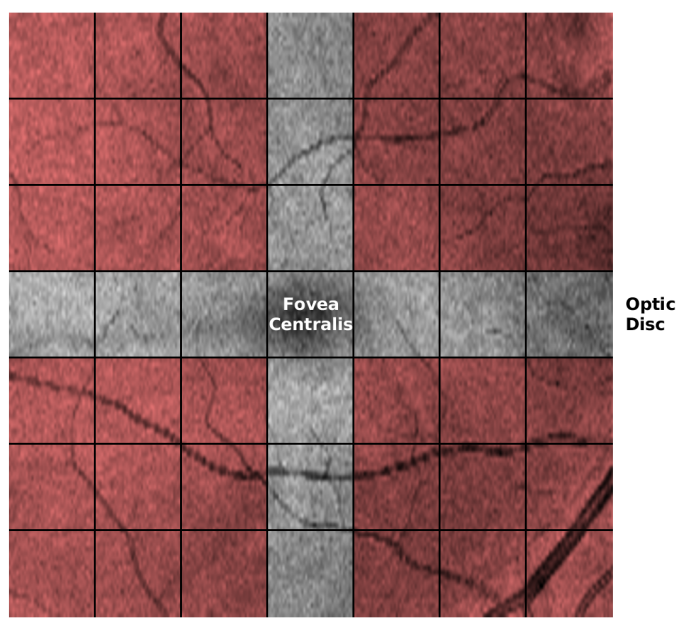


The DTCWT method was applied to the full macular area covered by the MVF image. The variance of the magnitude of the DTCWT complex coefficients was computed for six image subbands $\left( \pm 15^{\circ}, \pm 45^{\circ}\right.$ and $\left.\pm 75^{\circ}\right)$, as described in [5134]. These six variance values were used as the global features from each MVF image.

In total, 86 texture features were computed from each MVF of each of the six retinal layers being considered: 80 (20 × 4 quadrants) local GLCM-based features, and six global DTCWT-based features.

\subsection{MVF image computation methods}

Let $I_{A}$ and $I_{B}$ be the average intensity values (of a particular layer of the retina) from two A-scans located at two neighbour locations $A$ and $B$ that will produce two pixels of the corresponding fundus image (the MVF [14]). For our demonstration, we assume that $I_{B}=I_{A}+\Delta I$. Also, let $t_{A}$ and $t_{B}$ be the number of samples within the layer (thickness) in the positions of the considered A-scans, with $t_{A} \approx t_{B}$ (because these are side-by-side A-scans). Let $I_{M}$ e $I_{m}$, respectively, be the maximum and minimum intensity values in a block where the GLCM is to be computed. Finally, let us consider $N$ the number of grey-levels considered to compute the GLCM, and $K$ the total number of samples in an A-scan. We will illustrate that the alternative method to compute a MVF image considering the average over the entire A-scan has a more discriminative power to small differences in intensity $(\Delta I)$, except for the case where the thickness is constant across the GLCM block where both methods will produce the same results.

\section{The original MVF computation method}

The intensity amplitude within the GLCM block, as given by

$$
h_{o}=\frac{I_{M}-I_{m}}{N}
$$

Therefore, the level within the greyscale for $I_{A}$ and $I_{B}$ is given by

$$
\alpha_{o}\left(I_{A}\right)=\frac{I_{A}-I_{m}}{h_{o}} \quad \text { and } \quad \alpha_{o}\left(I_{B}\right)=\alpha_{o}\left(I_{A}+\Delta I\right)=\alpha_{o}(A)+\frac{\Delta I}{h_{o}},
$$

where the mapping of computed intensities to the individual greyscale levels (from 0 to $\mathrm{N}-1$ ) is given by $\lfloor\alpha()$.$\rfloor , and \lfloor$.$\rfloor is the floor operation. Therefore, the$ difference between the two intensity levels $\left(I_{A}\right.$ and $\left.I_{B}\right)$ is given by

$$
\Delta \alpha_{o}=\frac{\Delta I}{h_{o}} .
$$

The alternative MVF computation method 
In this case, using the same notation, the new intensity values are given by $\left(I_{A} t_{A}\right) / K$ and $\left(I_{B} t_{B}\right) / K$. Similarly, one defines the intensity amplitude within the GLCM block as

$$
h_{a}=\frac{\max (I t)-\min (I t)}{N K} \leq \frac{I_{M} \max (t)-I_{m} \min (t)}{N K} .
$$

Then one gets the level in greyscale for the computation of the GLCM given by

$$
\alpha_{a}\left(I_{A}\right)=\frac{I_{A} t_{A}-I_{m} \min (t)}{K h_{a}}
$$

and, by straightforward computations,

$$
\alpha_{a}\left(I_{B}\right)=\frac{\left(I_{A} t_{A}-I_{m} \min (t)\right)+\Delta I t_{A}+\Delta t I_{B}}{K h_{a}}=\alpha_{a}(A)+\frac{\Delta I t_{A}+\Delta t I_{B}}{K h_{a}},
$$

with $\Delta t=t_{B}-t_{A}$ and $\Delta I=I_{B}-I_{A}$, which means that the difference in greyscale is given by

$$
\Delta \alpha_{a}=\frac{\Delta I t_{A}+\Delta t I_{B}}{K h_{a}} .
$$

\section{Comparison between the discriminant powers}

In the original method, $I_{A}$ and $I_{B}$ are assigned the levels of, respectively, $\alpha_{o}\left(I_{A}\right)$ and $\alpha_{o}\left(I_{B}\right)$, which differ by $\Delta I / h_{o}$. On the other hand, for the alternative method, the difference in assigned levels is $\left(\Delta I t_{A}+\Delta t I_{B}\right) /\left(K h_{a}\right)$, being the latter higher than the former. One can compute the ratio $(\gamma)$ of the differences and show that this ratio is over the unit, meaning that the latter approach presents a better discriminative power.

$$
\gamma=\frac{\frac{\Delta I t_{A}+\Delta t I_{B}}{K h_{a}}}{\frac{\Delta I}{h_{o}}}=\frac{h_{o}}{h_{a} K}\left(t_{A}+\frac{\Delta t I_{B}}{\Delta I}\right) .
$$

Therefore one has from (1)

$$
\frac{h_{o}}{h_{a} K}=\frac{\frac{I_{M}-I_{m}}{N}}{K \frac{\max (I t)-\min (I t)}{N K}} \geq \frac{I_{M}-I_{m}}{I_{M} \max (t)-I_{m} \min (t)} \approx \frac{1}{\bar{t}}
$$

with $\bar{t}$ the average thickness within the GLCM block.

From (2) and (3), one gets the approximate lower bound for $\gamma$ as

$$
\gamma \gtrsim \underbrace{\frac{t_{A}}{\bar{t}}}_{\approx 1}+\frac{\Delta t I_{B}}{\bar{t} \Delta I} \gtrsim 1+\frac{\Delta t}{\bar{t}} \cdot \frac{I_{B}}{\Delta I} .
$$

The lower bound for $\gamma$, as defined above, is based on the assumption that the amplitude in thickness within a GLCM blocks is small - which verifies for 
the block divisions used (see figures 2 and 3 ), hence the ratio between $t_{A}$ and the average thickness is close to 1 - and differences in image intensity across two neighbour pixels is small in comparison to their average value - which verifies in general except for lower intensity image regions, in which case both methods will map to the same greyscale level, therefore, producing the same result.

Fig. 3. Cropped OCT B-scan image from the right eye of male subject of the younger group. This B-scan crosses the third and fourth quadrants (Q3 and Q4) and shows the limits for the GLCM blocks in white solid vertical lines. The central block $\left(4^{\text {th }}\right)$ is not used in our analysis (see figure 2). Red lines show the segmentation of the different layers of the retina as computed by the method used 20111]. Image axes are: x-axis (horizontal) - temporal (left) to nasal (right) and z-axis (vertical) anterior (vitreous top) to posterior (choroid - bottom).

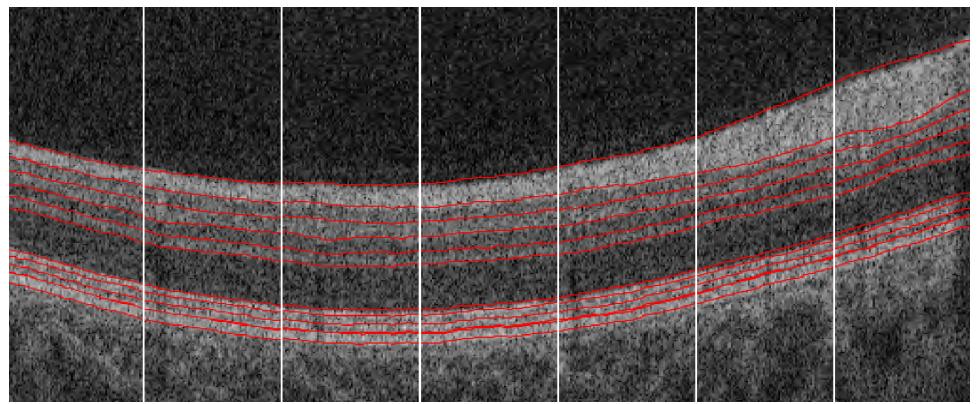

As shown, the discriminative power of the alternative method is superior to that of the original method for small differences between neighbour pixel intensities, which is the default scenario in healthy or close to the health condition retinas. Moreover, it is also clear that both approaches have similar results in the cases of small variations in thickness for a given layer.

\subsection{Statistical analysis}

All texture features were tested for normality using the Kolmogorov-Smirnov test ( $10 \%$ significance level for a conservative normality decision). For each feature, when both the female and the male groups followed a normal distribution, the two-sample t-test was used to test mean differences. Otherwise, the MannWhitney U-test (non-parametric) was used. The obtained p-values were separated into three significance levels: $p \leq 0.05, p \leq 0.01$ and $p \leq 0.001$. 


\section{Results}

The first analysis concerns the younger females and males in the present study. Table 2 shows the list of texture features presenting statistically significant differences between the two younger groups. Differences between the younger female and male groups are shown using the symbols $\mathbf{O}, \mathbf{a}$, and $\boldsymbol{*}$, and can be found in every single layer. The highest statistical differences can be found in the IPL (local GLCM features) and the GCL, INL and ONL (global DTCWT features). In the INL, texture differences are spread the most, as they cover three consecutive quadrants. Q2, the nasal-superior quadrant, holds consistent differences at consecutive layers of the retina - from the IPL to the ONL, while the same occurs for Q3, the temporal-inferior quadrant, from the GCL to the INL.

The second analysis concerns the older females and males. Overall, a minimal number of statistically significant differences was found between the two older groups: only two local features in the GCL, and three local features the ONL, all at the $5 \%$ significance level (shown in square-brackets - table 2 ).

\section{Discussion}

In order to reach a better understanding of the effect of gonadal hormones in different physiological and pathophysiological mechanisms, research, where the menopausal transition is taken into consideration, is particularly valuable, as it leverages the natural interruption of the production of the female hormones. Estrogen, for instance, is believed to have a major role in the sexual dimorphism observed not only in retinal disorders [26] but also in neurodegenerative diseases such as AD 28. Since the study of retinal biomarkers for disorders like AD using the retina as a window to the CNS has been gaining momentum in recent years 2133, understanding the interactions of estrogen and other gonadal hormones in the physiological mechanisms taking place in the retina, both pre- and postmenopause, is of utmost importance.

The main goal of the present work is a more in-depth exploration of the sexbased differences that were previously identified in the retinas of healthy adult subjects [23. For this purpose, we studied a larger population than the one used before [23] and performed two separate age-group analyses that excluded the probable perimenopause period of the women in the present study, in order to have pre- and postmenopause sex group comparisons.

Our results reveal numerous texture differences in all retinal layers between the two younger groups, which almost disappear in the older groups. These results suggest that texture analysis is an adequate tool to identify sex-based differences in the retina since it highlights them before the menopause when sexual dimorphism is more pronounced. Furthermore, it reveals that the observed sex-related differences become negligible after women reach menopause when gonadal hormones eventually stabilise at their permanent postmenopausal levels [29]. 
Table 2. Texture features presenting statistically significant differences between the female and male subjects for the retinal nerve fibre layer (RNFL); ganglion cell layer (GCL); inner plexiform layer (IPL); inner nuclear layer (INL); outer plexiform layer (OPL), and; outer nuclear layer (ONL). Hyphens (-) represent a $p$-value $>0.05$ (non-significant statistical difference), the green-coloured circles (O) represent a $p$ value $\leq 0.05$, the orange-coloured squares $(\square)$ represent a $p$-value $\leq 0.01$ and the red-coloured asterisks $(*)$ represent a $p$-value $\leq 0.001$ for the younger groups. Squarebrackets ([ ]) denote differences found between the older female and male groups (all at the $5 \%$ significance level). Q1 - Superior-temporal quadrant, Q2 - Superior-nasal quadrant, Q3 - Inferior-temporal quadrant and Q4 - Inferior-nasal quadrant. IDN stands for Inverse Difference Moment Normalized; in the features IMC1 and IMC2, IMC stands for Information Measure of Correlation; and INN stands for Inverse Difference Normalized.

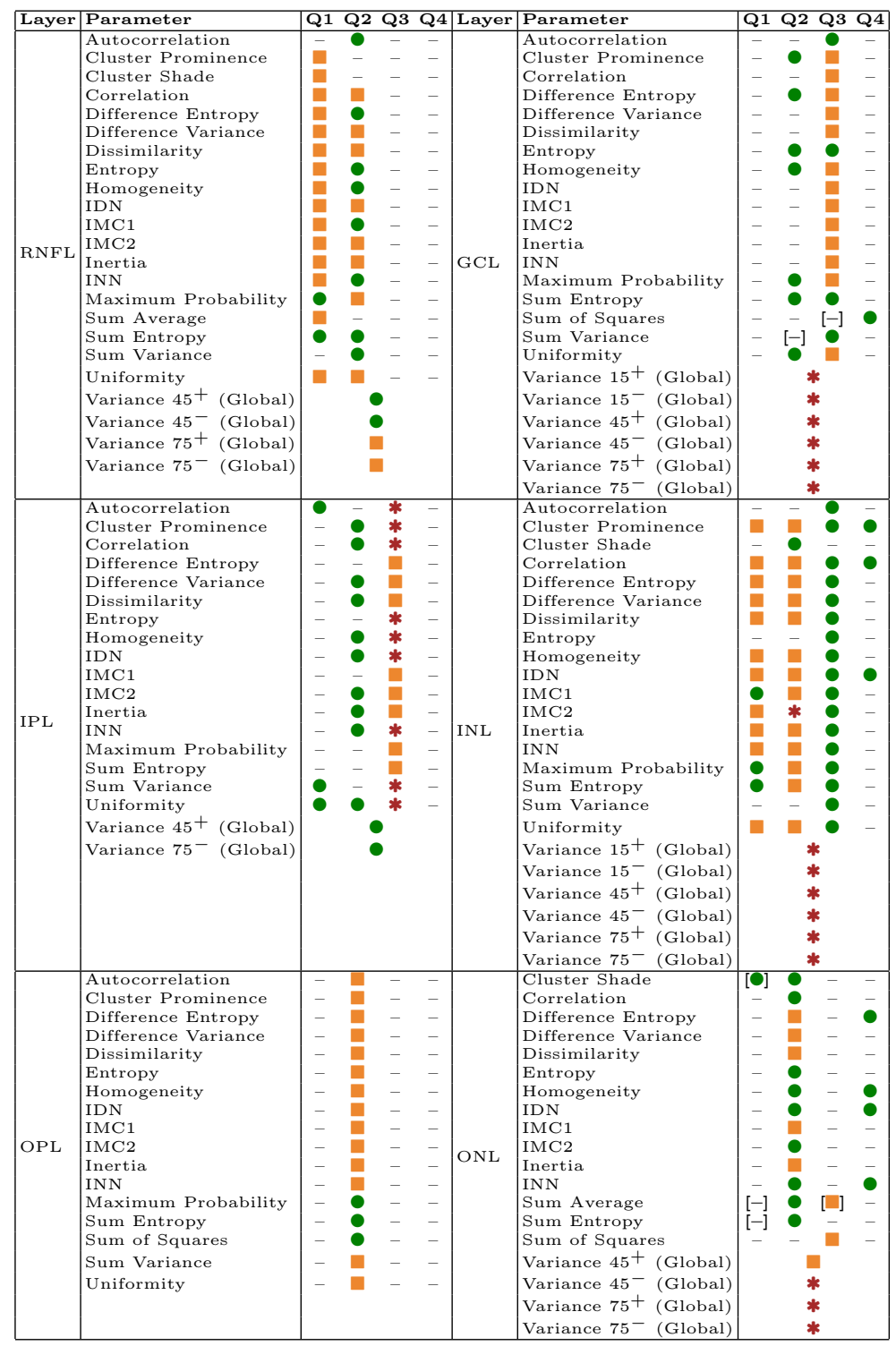


These results add to those from 23] where the menopause transition period was not taken into consideration. The results from that ([23]) menopauseindependent study showed numerous statistically significant differences between the female and male groups. In the present study, the differences across the two age group-analyses are very noticeable. The contrast in the number and significance of the sex-based differences across the younger and older groups supports the hypothesis that the differences across the younger and older groups can be correlated to menopause-based hormonal alterations.

Our group has been studying texture-based methods to extract new information from retinal fundus images computed from OCT data. Preliminary analyses suggested the MVF computation method herein presented may be more sensitive to differences present in the retina than the original method [14].

One limitation of the work herein reported is the fact that it is a retrospective study, which prevented the authors from collecting information regarding the phase of the menstrual cycle and the onset of menopausal transition for any of the females in the present study. The age of menopause has been defined as the age at the last menstrual period, which can only be determined retrospectively after a woman has stopped menstruating for 12 consecutive months [4. The authors believe that the used reference mean age of menopause (51 years old) is reasonably accurate, as most studies focusing on the menopausal transition are longitudinal [12, and so there is little risk of distortion on the reported timing of the last menstruation.

Nevertheless, in the present study, the older female group's lower age limit (54 years old) is reasonably close to the reference age of menopause (51 years old), so the youngest women in this group may be still menstruating. However, these women are most likely already going through the menopause transition period. Nevertheless, the limit of 54 years old was chosen to include the largest possible number of female/male pairs to populate both older groups, from all healthy control subjects in the authors' institutional database. Concerning the younger females' group (upper age limit of 42 years old), it is unlikely that the oldest women are already going through the menopausal transition, assuming that none of them had premature menopause ( $<40$ years) 37. For reference, Bromberger [4] used 47.5 years as the approximate age over which females would presumably be close to the time of menopause.

\section{Conclusion}

The present study confirms the existence of sex-based differences in the human retina, as measured by texture analysis of MVF images computed from OCT data. In this study, we first provide evidence suggesting that the neuroretina, the only part of the CNS directly accessible through optical means, presents distinct texture characteristics for premenopausal females and age-matched males and that differences almost disappear after women have gone through the menopausal period. Second, we apply a modified MVF image computation method that fur- 
ther highlights the retinal texture differences found.

\section{Conflict of interest}

The authors declare that they have no conflict of interest.

\section{References}

1. Abràmoff, M.D., Garvin, M.K., Sonka, M.: Retinal imaging and image analysis. IEEE Reviews in Biomedical Engineering 3, 169-208 (2010). https://doi.org/10.1109/RBME.2010.2084567

2. Adhi, M., Aziz, S., Muhammad, K., Adhi, M.I.: Macular thickness by age and gender in healthy eyes using spectral domain optical coherence tomography. PLoS ONE 7(5), e37638 (2012). https://doi.org/10.1371/journal.pone.0037638

3. Anantrasirichai, N., Achim, A., Morgan, J.E., Erchova, I., Nicholson, L.: SVMbased texture classification in optical coherence tomography. In: IEEE 10th International Symposium on Biomedical Imaging: from Nano to Macro. pp. 1332-1335 (2013). https://doi.org/10.1109/ISBI.2013.6556778

4. Bromberger, J.T., Matthews, K.A., Kuller, L.H., Wing, R.R., Meilahn, E.N., Plantinga, P.: Prospective study of the determinants of age at menopause. American Journal of Epidemiology 145(2), 124-133 (1997). https://doi.org/10.1093/oxfordjournals.aje.a009083

5. Celik, T., Tjahjadi, T.: Multiscale texture classification using dual-tree complex wavelet transform. Pattern Recognition Letters 30, 331-339 (2009). https://doi.org/10.1016/j.patrec.2008.10.006

6. Chan, A., Duker, J.S., Ko, T.H., Fujimoto, J.G., Schuman, J.S.: Normal macular thickness measurements in healthy eyes using stratus optical coherence tomography. Archives of Ophthalmologyy 124(2), 193-198 (2006). https://doi.org/10.1001/archopht.124.2.193

7. Clausi, D.A.: An analysis of co-occurrence texture statistics as a function of grey level quantization. Canadian Journal of Remote Sensing 28(1), 45-62 (2002). https://doi.org/10.5589/m02-004

8. Conners, R.W., Trivedi, M.M., Harlow, C.A.: Segmentation of a highresolution urban scene using texture operators ( Sunnyvale, California). Computer Vision, Graphics, \& Image Processing 25(3), 273-310 (1984). https://doi.org/10.1016/0734-189x(84)90197-x

9. Çubuk, M., Kasm, B., Koçluk, Y., Sukgen, E.A.: Effects of age and gender on macular thickness in healthy subjects using spectral optical coherence tomography / scanning laser ophthalmoscopy. International Ophthalmology 38, 127-131 (2018). https://doi.org/10.1007/s10792-016-0432-z

10. Ferreira, H., Serranho, P., Nunes, A., Moreira, P.I., Castelo-Branco, M., Ambrósio, A.F., Bernardes, R.: Characterization of the retinal changes of the 3xTg-AD mouse model of Alzheimer's disease. In: Henriques, J. (ed.) MEDICON 2019, IFMBE. vol. 76, pp. 1816-1821 (2020). https://doi.org/10.1007/978-3-030-31635-8 
The effect of menopause on the sexual dimorphism in the human retina

11. Garvin, M.K., Abràmoff, M.D., Wu, X., Russell, S.R., Burns, T.L., Sonka, M.: Automated 3-D intraretinal layer segmentation of macular spectral-domain optical coherence tomography Images. In: IEEE Transactions on Medical Imaging. vol. 28, pp. 1436-1447 (2009). https://doi.org/10.1109/TMI.2009.2016958

12. Gold, E.B.: The timing of the age at which natural menopause occurs. Obstetrics and Gynecology Clinics of North America 38(3), 425-440 (2011). https://doi.org/10.1016/j.ogc.2011.05.002

13. Guedes, V., Schuman, J.S., Hertzmark, E., Wollstein, G., Correnti, A., Mancini, R., Lederer, D., Voskanian, S., Velazquez, L., Pakter, H.M., Pedut-Kloizman, T., Fujimoto, J.G., Mattox, C.: Optical coherence tomography measurement of macular and nerve fiber layer thickness in normal and glaucomatous human eyes. Ophthalmology 110(1), 177-189 (2003). https://doi.org/10.1016/s0161-6420(02)01564-6

14. Guimarães, P., Rodrigues, P., Lobo, C., Leal, S., Figueira, J., Serranho, P., Bernardes, R.: Ocular fundus reference images from optical coherence tomography. Computerized Medical Imaging and Graphics 38, 381-389 (2014). https://doi.org/10.1016/j.compmedimag.2014.02.003

15. Haralick, R.M.: Statistical and structural approaches to texture. Proceedings of the IEEE 67(5), 786-804 (1979). https://doi.org/10.1109/PROC.1979.11328

16. Haralick, R.M., Shanmugam, K., Dinstein, I.: Texture features for image classification. In: IEEE Transaction on Systems, Man and Cybernetics. vol. SMC-3, pp. 610-621 (1973). https://doi.org/10.1109/TSMC.1973.4309314

17. Kashani, A.H., Zimmer-Galler, I.E., Shah, S.M., Dustin, L., Do, D.V., Eliott, D., Haller, J.A., Nguyen, Q.D.: Retinal thickness analysis by race, gender, and age using stratus OCT. American Journal of Ophthalmology 149, 496-502 (2010). https://doi.org/10.1016/j.ajo.2009.09.025

18. Kassner, A., Thornhill, R.E.: Texture Analysis: a review of neurologic MR imaging applications. American Journal of Neuroradiology 31, 809-816 (2010). https://doi.org/10.3174/ajnr.A2061

19. Kelty, P.J., Payne, J.F., Trivedi, R.H., Kelty, J., Bowie, E.M., Burger, B.M.: Macular thickness assessment in healthy eyes based on ethnicity using stratus OCT optical coherence tomography. Investigative Ophthalmology and Visual Science 49(6), 2668-2672 (2008). https://doi.org/10.1167/iovs.07-1000

20. Li, K., Wu, X., Chen, D.Z., Sonka, M.: Optimal surface segmentation in volumetric images - a graph-theoretic approach. In: IEEE Transactions on Pattern Analysis and Machine Intelligence. vol. 28, pp. 119-134 (2006). https://doi.org/10.1109/TPAMI.2006.19

21. London, A., Benhar, I., Schwartz, M.: The retina as a window to the brain - from eye research to CNS disorders. Nature Reviews Neurology (2012). https://doi.org/10.1038/nrneurol.2012.227

22. Nunes, A., Ambrósio, A.F., Castelo-Branco, M., Bernardes, R.: Texture biomarkers of Alzheimer's disease and disease progression in the mouse retina. In: 2018 IEEE 18th International Conference on Bioinformatics and Bioengineering. pp. 41-46 (2018). https://doi.org/10.1109/BIBE.2018.00016

23. Nunes, A., Serranho, P., Quental, H., Ambrósio, A.F., Castelo-Branco, M., Bernardes, R.: Sexual dimorphism of the adult human retina assessed by optical coherence tomography. In: Henriques, J. (ed.) MEDICON 2019, IFMBE. vol. 76, pp. 1830-1834 (2020). https://doi.org/10.1007/978-3-030-31635-8

24. Nunes, A., Silva, G., Alves, C., Batista, S., Sousa, L., Castelo-Branco, M., Bernardes, R., Bernardes, R.: Textural information from the retinal nerve fibre layer in multiple sclerosis. In: 2019 IEEE 6th Portuguese Meeting on Bioengineering (ENBENG) (2019). https://doi.org/10.1109/ENBENG.2019.8692454 
25. Nunes, A., Silva, G., Duque, C., Januário, C., Santana, I., Ambrósio, A.F., CasteloBranco, M., Bernardes, R.: Retinal texture biomarkers may help to discriminate between Alzheimer's, Parkinson's, and healthy controls. PLoS ONE 14(6), e0218826 (2019). https://doi.org/10.1371/journal.pone.0218826

26. Nuzzi, R., Scalabrin, S., Becco, A., Panzica, G.: Gonadal hormones and retinal disorders: a review. Frontiers in Endocrinology 9(66) (2018). https://doi.org/10.3389/fendo.2018.00066

27. Ooto, S., Hangai, M., Tomidokoro, A., Saito, H., Araie, M., Otani, T., Kishi, S., Matsushita, K., Maeda, N., Shirakashi, M., Abe, H., Ohkubo, S., Sugiyama, K., Iwase, A., Yoshimura, N.: Effects of age, sex, and axial length on the threedimensional profile of normal macular layer structures. Investigative Ophthalmology and Visual Science 52, 8769-8779 (2011). https://doi.org/10.1167/iovs.11-8388

28. Ratnakumar, A., Zimmerman, S.E., Jordan, B.A., Mar, J.C.: Estrogen activates Alzheimer's disease genes. Alzheimer's \& Dementia: Translational Research \& Clinical Interventions 5, 906-917 (2019). https://doi.org/10.1016/j.trci.2019.09.004

29. Santoro, N.: Perimenopause: from research to practice. Journal of Women's Health 25(4), 332-339 (2016). https://doi.org/10.1089/jwh.2015.5556

30. Selesnick, I.W.W., Baraniuk, R.G.G., Kingsbury, N.C.C.: The dual-tree complex wavelet transform. IEEE Signal Processing Magazine pp. 123-151 (2005). https://doi.org/10.1109/MSP.2005.1550194

31. Soh, L.., Tsatsoulis, C.: Texture analysis of sar sea ice imagery using gray level cooccurrence matrices. IEEE Transactions on Geoscience and Remote Sensing 37(2), 780-795 (March 1999). https://doi.org/10.1109/36.752194

32. Song, W.K., Lee, S.C., Lee, E.S., Kim, C.Y., Kim, S.S.: Macular thickness variations with sex, age, and axial length in healthy subjects: a spectral domain-optical coherence tomography study. Investigative Ophthalmology and Visual Science 51, 3913-3918 (2010). https://doi.org/10.1167/iovs.09-4189

33. Svetozarskiy, S.N., Kopishinskaya, S.V.: Retinal optical coherence tomography in neurodegenerative diseases (Review). Sovremennye Tehnologii v Medicine 7(1), 116-123 (2015). https://doi.org/10.17691/stm2015.7.1.14

34. Wang, S., Lu, S., Dong, Z., Yang, J., Yang, M., Zhang, Y.: Dual-tree complex wavelet transform and twin support vector machine for pathological brain detection. Applied Sciences 6(169), 1-18 (2016). https://doi.org/10.3390/app6060169

35. Wong, A., Chan, C., Hui, S.: Relationship of gender, body mass index, and axial length with central retinal thickness using optical coherence tomography. Eye 19, 292-297 (2005). https://doi.org/10.1038/sj.eye.6701466

36. World Medical Association: World Medical Association Declaration of Helsinki: ethical principles for medical research involving human subjects. Journal of the American Medical Association 310(20), 2191-2194 (2013). https://doi.org/10.1001/jama.2013.281053

37. Zhu, D., Chung, H.f., Dobson, A.J., Pandeya, N., Giles, G.G., Bruinsma, F., Brunner, E.J., Kuh, D., Hardy, R., Avis, N.E., Gold, E.B., Derby, C.A., Matthews, K.A., Cade, J.E., Greenwood, D.C., Demakakos, P., Brown, D.E., Sievert, L.L., Anderson, D., Hayashi, K., Lee, J.S., Mizunuma, H., Tillin, T., Simonsen, M.K., Adami, H.O., Weiderpass, E., Mishra, G.D.: Age at natural menopause and risk of incident cardiovascular disease: a pooled analysis of individual patient data. Lancet Public Health 4, 553-564 (2019). https://doi.org/10.1016/S2468-2667(19)30155-0 